\title{
A Method of Information Resource Classification and Coding for Implementing Smart Residing Application System
}

\author{
Qingchun Jiao, a , Guangping Zhou ${ }^{2, b}$ and Ning $\mathrm{Li}^{3, \mathrm{c}}$ \\ ${ }^{1}$ School of Automation \& Electrical Engineering, Zhejiang University of Science \& Technology, \\ Hangzhou 313000, China; \\ ${ }^{2}$ School of Information \& Electronic Engineering, Zhejiang University of Science \& Technology, \\ Hangzhou 313000, China. \\ ${ }^{3}$ Standardization Research Institute of Zhejiang Province, Hangzhou 313000, China. \\ a113030@zust.edu.cn, b104026@zust.edu.cn, '147821034@qq.com
}

Keywords: Smart residing, Information classification, Information coding.

\begin{abstract}
Information classification and coding is the fundamental work of informatization. It is an important way to solve difficult issues in information system construction such as non-unified information format, information sharing and exchange problems. After analyzing the information classification and coding research progress, based on the current developing of smart residing, an information sharing oriented smart residing information classification and coding system was proposed in this paper and the coding process was illustrated. The results of this study will help to promote the smart residing informatization, and improve the level of exchanging and sharing of smart residing information resources.
\end{abstract}

\section{Introduction}

Combined with the various demand of smart city developing, smart residing application system is such a business system which can solve all kinds of business needs. It is a representative comprehensive smart application implementing smart service, smart management and smart living which can make the various big data coming from smart city general support platform be utilized comprehensively and intelligently[1]. Smart residing application system includes livable residential services such as information service and residential business, regional security function like video monitoring, event management and control, access control, video intercom, visitor management, parking management and traffic management. In the process of building smart residing application system, the development and utilization of information resources is the core content. It is necessary to carry out information classification and coding standardization work to fully utilizing these information resources. If the research of information classification and coding limited to a certain area, there will be "information island" phenomenon, therefore, the overall situation should be considered to establish a unified, sharing oriented classification and coding information system, make all kinds of scattered and isolated information resources into network sharing information resources, integrate numerous "island style" information systems, achieve information sharing and exchange [2].

Information classification and coding is the basis of information storage, processing, exchange and sharing [3], it is an important technical foundation and premise to establish a variety of information management system. Unified Information classification and coding could achieve the integration and standardization of names, terminology and coding, and guide the building of information systems and databases. Implementing the Information classification and coding of smart residing could harmonize capturing and processing of various departments' information resources to meet the overall requirements of smart residing application system and the business needs of smart residing units. Meanwhile, it could reduce the duplication of information resource collection, processing, sorting and storage, best eliminate the misunderstandings and disagreements of information resource naming and describing due to the classification and coding inconsistencies, 
avoid the confusion such as multiple names for one object, one name for multiple objects or different classification and coding for the same information content.

Information classification and coding is a procedure of classifying information scientifically and systematically and assigning with a specific code. The quality and efficiency of information sharing and exchange between the various information systems will be affected directly by the quality of information classification and coding structure design [4]. Therefore, develop a unified smart residing information classification and coding system, eliminate the confusion in full measure caused by the inconsistence of smart residing resource pool information naming, description, classification and coding, represent the smart residing basic resource information in a unified way, is an important prerequisite to achieve smart residing.

\section{Smart Residing Information Resources System}

\subsection{Basic Principles.}

The section headings are in boldface capital and lowercase letters. Second level headings are typed as part of the succeeding paragraph (like the subsection heading of this paragraph). All manuscripts must be in English, also the table and figure texts, otherwise we cannot publish your paper. Please keep a second copy of your manuscript in your office. When receiving the paper, we assume that the corresponding authors grant us the copyright to use the paper for the book or journal in question. When receiving the paper, we assume that the corresponding authors grant us the copyright to use the paper for the book or journal in question. When receiving the paper, we assume that the corresponding authors grant us the copyright to use.

The specification "The basic principles and methods for information classifying and coding" (GB7027-2002) was published by government for information classification and coding standardization, which defined some concepts of information classification and coding, introduced the basic principles and methods for information classification and coding. Accordingly, classifying the smart residing information resources should adhere to the following principles:

1) Scientificity. Choose the most stable natural attributes or characteristics of smart residing information resources as the foundation for classifying;

2) Systematicity. Systemize the selected classification object attributes or characteristics of smart residing information resources according to a certain order, to form a rational and scientific classification system;

3) Expandability. Category setting could ensure that the established classification system will not be disrupted when new classification object of smart residing information resources added, meanwhile expanding could also be achieved on the basis of the classification system;

4) Practicability. Classifying smart residing information resources should meet the actual needs of the relevant units within the system in terms of the system engineering as far as possible, under the premise of meeting the overall system tasks and requirements.

\subsection{Basic Methods.}

The most common basic methods in information classification are linear classification, side classification and mixed classification. Wherein the line classification method, also known as hierarchical classification or systematic classification; side classification method is also known as faceted classification.

Linear classification [5] is based on certain characteristics or properties of objects to classify categories, level by level refine categories, expand the category sections gradually, and form the hierarchical and tree shape classification system. The standards using linear classification such as GB/T 18317-2001, thematic map information classification and code, GB/T13923-2006, fundamental geographic information features classification and code, and GB/T 13745-2008, subject classification and Code.

Side classification is based on the various inherent characteristics or properties of objects to classify categories, divided them into separate sides, each contains a set of categories, there is no affiliation between the categories, and combine the different categories in to form a new composite 
category. The promulgated standard GB 12403-90, Official Positions Title Code, is using side classification.

Mixed classification refers to the classification of linear and side classification used in combination with one as mainly another as supplementary.

\subsection{Classification System.}

According to the above classification principles, combined with smart residing information resources characteristics, the smart residing information classification system was developed by linear classification. The classification system is divided into two main categories, consists of 15 sub-categories in total, and could be expanded as needed, each category could be broken down (Fig. 1). The specific characteristics of each category information are as follows.

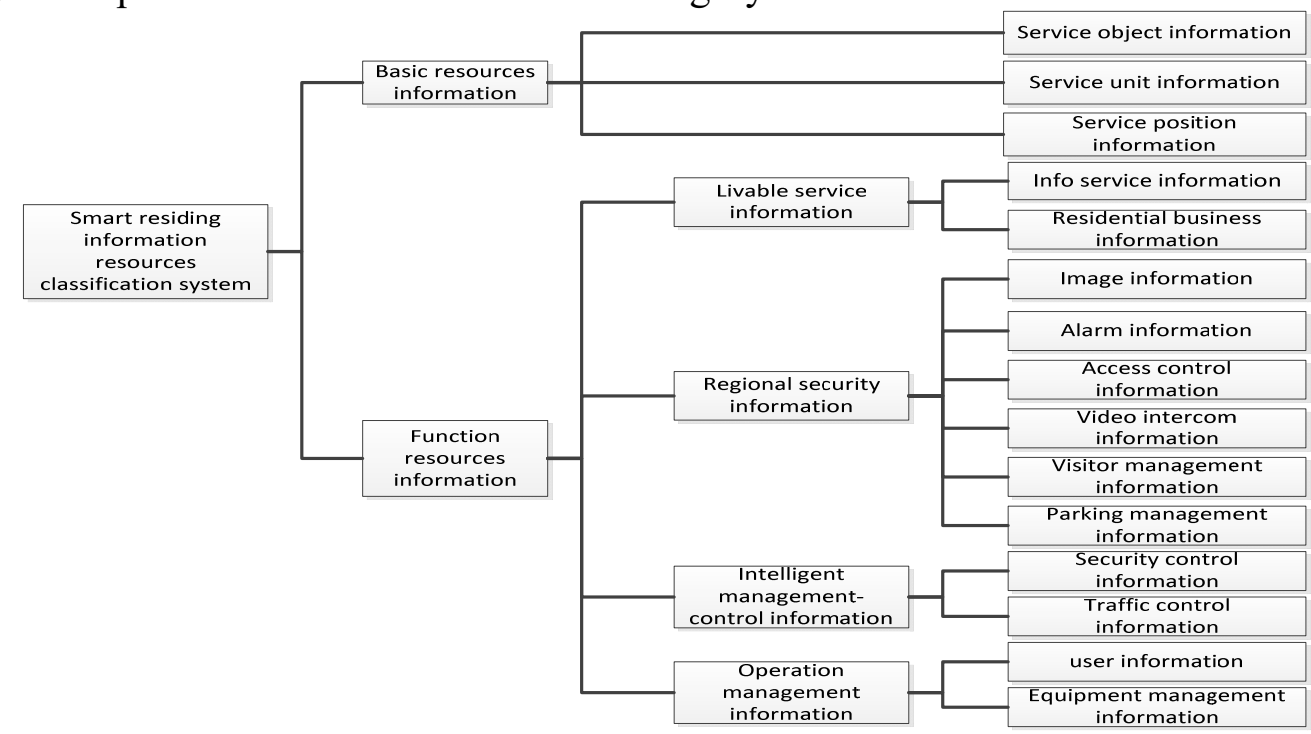

Fig. 1 Smart residing information resources classification system

Smart residing information resources are mainly comprised of basic information resources and function information resources. Basic Information resource refers to all types of information resources with smart residing services stakeholders, including service objects (people, house, vehicle, public facilities), service units (security service companies, health services, housing maintenance, elevator maintenance, etc.), services spatial location, etc.

Function information resource refers to all types of information resources provided by the smart residing application system, including four categories such as livable service information, regional security information, intelligent management and control information and operation and maintenance management information.

Livable service information refers to efficient, convenient and personalized information services and residential business social service information provided for residents within the region. Information service information includes initiative pushing public information, customized lifestyle information service information which residents selected. Residential business information includes housekeeping information, subscription information, event management information and business yellow pages information.

Regional security information is a variety of information resources related to security management, including various types of image information, alarms, access control, video intercom, visitor management and parking management and so on. Intelligent management refers to security management control information and traffic control information. Operation and maintenance management information refers to a variety of information resources related to operation and maintenance management functions, including various types of user information management, equipment management, video diagnostics, statistical analysis and fault repair and so on. 


\section{Smart Residing Information Resources Classification Coding}

\subsection{Basic Principles.}

To meet the information objects identification, information resource classification coding of smart residing application system should adhere to the following principles $[6,7]$ :

1) Uniqueness. Each coding object can only have a code, one code only expresses a unique coding object.

2) Rationality. Coding structure should adapt to classification system.

3) Extendibility. The expanding demand for similar coding could be satisfied, in order to add new coding objects without disorganizing the original architecture and have sufficient expansion capacity.

4) Compatibility. Harmonized with relevant standards (including international standards, national standards, and industry standards).

5) Efficiency. Coding structure should be as simple as possible, which saves storage space and easy to process by computer efficiently.

6) Applicability. The code should reflect the characteristics of coding object as far as possible, helpful to remember and convenient to encode.

\subsection{Code Structure.}

Smart residing information resource classification code consists of two parts which separated by the character "." (Code position 6). The preceding five bits are the first part which represent smart residing category code. The category code is comprised of three characters and two digits. The two digits (Code position $4 \& 5$ ) represent sub-category code arranged in ascending number sequence starting from 01 and reserved appropriately for expanding code.

The second part is extended identity code. The first two bits (code position $7 \& 8$ ) of extended identity code represent information attribute and the following bits are variable length identifier. The information attribute " 00 " indicates that the following variable length identifier is smart residing user-defined code, subsequent numbers from " 01 " correspond to the relevant international standards. If the position 7, 8 are all 0 , then the following bits are two digital user-defined code (code position 9 $\& 10$ ). If the position 7,8 are not all 0 , then the following bits are variable length external reference code which reference to the corresponding standard code directly. The code format is described as follows:

Code structure: XXX XX . XX XXX

Example:

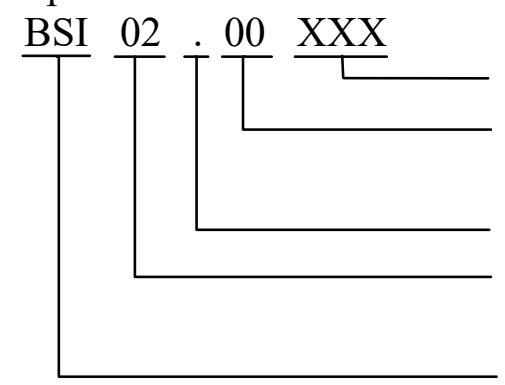

Variable length identifier

2 bits refernce identifier( 00 represents the following

User-defined code of this standard)

Separator

Information sub-category code(Arabic numerals, use 0 at complement bit)

3 bits category code( the first bit is B or F which represent Basic Or Functional Resource Information, the second and third bit are information category)

Fig. 2 Code description

\section{Implementation Example}

Smart residing application system personnel information, for example, each attribute code of personnel information are described in the table below. The letter B at the first bit position indicates "Basic Information" of information category, the following two letters SI indicate "Service Object 
Information" of basic resource information in information sub-category, the digits 02 at the position 4 $\& 5$ indicate number sequence of personnel information in basic resource information sub-category.

Following the seperator is the extended identity code, take the extended identity code " 010001 " for "citizen identity number" as example, the first two digits "01" indicate "GB/T 21062.3-2007 Government information resource interchange system-Part 3: Data interface specification", the following digits " 0001 " indicate the identifier of "citizen identity number" in the national standard "GB/T 21062.3-2007" is referenced.

In the extended identity code " 0007 " of the identifier code for "Zip Code", " 00 " indicates smart residing user-defined code, "07" is the sequence number for "Zip Code" in personnel information codes.

Table 1 Classification Code of Personnel Information

\begin{tabular}{|c|c|}
\hline Classification Code & Name \\
\hline BSI 02.01 0001 & Citizen identity number \\
\hline BSI 02.01 0002 & Name \\
\hline BSI 02.01 0003 & Gender \\
\hline BSI 02.01 0004 & Nation \\
\hline BSI 02.01 0005 & Date of Birth \\
\hline BSI 02.01 0006 & Address \\
\hline BSI 02.00 07 & Zip Code \\
\hline BSI 02.00 08 & Age \\
\hline BSI 02.00 09 & Mobile Phone Number \\
\hline BSI 02.00 10 & Email \\
\hline
\end{tabular}

\section{Conclusion}

Smart residing information resource classifying and coding is the fundamental work of smart residing project, and is the premise and basis for implementing smart residing information expressing, exchanging, sharing and information systems integrating. By describing, defining, classifying and encoding all kinds of information of smart residing, make them described by same format to solve the duplicate entering conflicts of same information in smart residing application systems caused by the non-uniform information code problem among industries and companies, exert the most integrated and economic benefits of smart residing application system. The suggested smart residing information resource classification and coding system in this paper take advantage of those international and domestic standards applied in related industries, make part of the coding could be referenced directly from other standards and not necessary to define again, therefore the efficiency of code reuse and sharing could be enhanced. However, the coding system of this paper is still in the exploratory stage, the specific content of each category need to be further refined and the system need to be improved in future work.

Project Source: AQSIQ nonprofit industry research project "City-oriented smart residing common technical standards developing and demonstration", project Number: 201310206; The research result "Smart city infrastructure evaluation system and evaluation model research" from Philosophy and Social key research base of Zhejiang Province, Industrial development policy research center of Zhejiang Province, project number: 14JDCY01Z.

\section{References}

[1]. Ning Li, Ziwei Chen, Ruowei Liu. Research on Standardization of Smart Residing System Application. Information Technology \& Standardization. (2015) No. 7, p. 41-44.

[2]. Fahui Gu, Wen Li, Luyan Lan. Study of the Information Sharing-Oriented Information Classification and Coding System and Implementation Process. Journal of Intelligence. (2008) No. 4 , p. 25-28. 
[3]. Maozhen Zhang, Tieying Song, Tang Xiaoming. Classification and coding of forest resources information. Journal of Fujian College of Forestry. Vol. 25(2005) No. 2, p. 147-152.

[4]. Fahui Gu: Study of the Information Sharing-Oriented Information Classification and Coding and Management System(Master, Jiangxi University of Science \& Technology, China, 2008). p. 10.

[5]. Qingsen Zeng, Hong Zhang, Wei Tian. The Research in the Classification and Coding of the Packaging Enterprise Information. Packaging Engineering. Vol. 21 (2000) No. 2, p. 37-39.

[6]. Changwei Yuan, Fahui Gu. Discussion on Information Classification and Coding. Science Mosaic. (2010) No. 5, p. 248-251.

[7]. Xiujian Xiao, Jiashun Wang, Tianmiao Wang, et al. Information Encoding and the Object-Oriented Information Code Model. Microcomputer Information. Vol 19 (2003) No. 6, p. 79-80. 